\title{
Erratum to: Many Body Interactions in Nuclear Matter
}

\author{
E. G. Drukarev ${ }^{a}$, M. G. Ryskin ${ }^{a}$, and V. A. Sadovnikova ${ }^{a, *}$ \\ ${ }^{a}$ National Reseach Center Kurchatov Institute, Konstantinov Nuclear Physics Institute, \\ Gatchina, Leningrad oblast, 188300 Russia \\ *e-mail: sadovnik@thd.pnpi.spb.ru \\ Submitted October 8, 2019; accepted for publication October 8, 2019
}

DOI: $10.3103 / \mathrm{S} 1062873819150012$

On page 1166, above Eq. (5), the sentence should read as follows:

Presenting $\kappa^{(1)}(\rho)=\kappa_{N}^{(1)}(\rho) \rho$ and $\left(\kappa_{N}+\kappa_{N}^{(1)}(\rho)\right)=\hat{\kappa}_{N}(\rho) \rho$ we find $\hat{\kappa}_{N}(\rho)=11.4$ at phenomenological value of the saturation density $\mathrm{r} 0=0.17 \mathrm{fm}^{-3}$.

The original article can be found online at https://doi.org/10.3103/S1062873819090041. 